\title{
Morbidity after elective surgery in patients on chronic dialysis: a systematic review and meta-analysis
}

Dharmenaan Palamuthusingam ${ }^{1,2,3^{*}}$, Arun Nadarajah ${ }^{4}$, David Wayne Johnson ${ }^{2,5,6}$, Elaine Marie Pascoe ${ }^{7}$, Carmel Marie Hawley ${ }^{2,5,7}$ and Magid Fahim ${ }^{5,7}$

\begin{abstract}
Background: Patients on chronic dialysis are at increased risk of postoperative mortality following elective surgery compared to patients with normal kidney function, but morbidity outcomes are less often reported. This study ascertains the excess odds of postoperative cardiovascular and infection related morbidity outcomes for patients on chronic dialysis.

Methods: Systematic searches were performed using MEDLINE, Embase and the Cochrane Library to identify relevant studies published from inception to January 2020. Eligible studies reported postoperative morbidity outcomes in chronic dialysis and non-dialysis patients undergoing major non-transplant surgery. Risk of bias was assessed using the Newcastle-Ottawa Scale and the certainty of evidence was summarised using GRADE. Random effects meta-analyses were performed to derive summary odds estimates. Meta-regression and sensitivity analyses were performed to explore heterogeneity.

Results: Forty-nine studies involving 10,513,934 patients with normal kidney function and 43,092 patients receiving chronic dialysis were included. Patients on chronic dialysis had increased unadjusted odds of postoperative cardiovascular and infectious complications within each surgical discipline. However, the excess odds of cardiovascular complications was attenuated when odds ratios were adjusted for age and comorbidities; myocardial infarction (general surgery, OR 1.83 95\% 1.29-2.36) and stroke (general surgery, OR 0.95, 95\%Cl 0.84-1.06). The excess odds of infectious complications remained substantially higher for patients on chronic dialysis, particularly sepsis (general surgery, OR 2.42, 95\% Cl 2.12-2.72).

Conclusion: Patients on chronic dialysis are at increased odds of both cardiovascular and infectious complications following elective surgery, with the excess odds of cardiovascular complications attributable to being on dialysis being highest among younger patients without comorbidities. However, further research is needed to better inform perioperative risk assessment.
\end{abstract}

Keywords: Perioperative morbidity, Chronic dialysis, Surgery, Infection, End-stage kidney failure

\footnotetext{
* Correspondence: Dharmenaan.palamuthusingam@health.qld.gov.au; menaan@gmail.com

${ }^{1}$ Metro South Integrated Nephrology and Transplant Services, Logan

Hospital, Armstrong Road \& Loganlea Road, Meadowbrook, Queensland

4131, Australia

${ }^{2}$ Faculty of Medicine, University of Queensland, Armstrong Road \& Loganlea

Road, St Lucia, Queensland 4072, Australia

Full list of author information is available at the end of the article
}

C C The Author(s). 2021 Open Access This article is licensed under a Creative Commons Attribution 4.0 International License, which permits use, sharing, adaptation, distribution and reproduction in any medium or format, as long as you give appropriate credit to the original author(s) and the source, provide a link to the Creative Commons licence, and indicate if changes were made. The images or other third party material in this article are included in the article's Creative Commons. licence, unless indicated otherwise in a credit line to the material. If material is not included in the article's Creative Commons licence and your intended use is not permitted by statutory regulation or exceeds the permitted use, you will need to obtain permission directly from the copyright holder. To view a copy of this licence, visit http://creativecommons.org/licenses/by/4.0/ The Creative Commons Public Domain Dedication waiver (http://creativecommons.org/publicdomain/zero/1.0/) applies to the data made available in this article, unless otherwise stated in a credit line to the data. 


\section{Introduction}

Advances in surgical techniques and perioperative care pathways have resulted in improved outcomes and facilitated access to surgery for increasingly complex patient cohorts, such as those with end-stage kidney disease (ESKD) [1]. A previous systematic review has shown that elective surgery in chronic dialysis patients is associated with a higher risk of postoperative mortality compared to patients with normal kidney function, which is at least partially due to a higher comorbid illness burden and older age [2-4]. Although death is the most serious complication, it is also an insensitive marker of cure or maintenance of function, and in fact qualitative research has shown that chronic dialysis patients prioritise maintenance of daily functional capacity over avoiding death itself $[5,6]$. Therefore, accurate assessment of the risks of non-fatal postoperative outcomes may facilitate more meaningful patient engagement in shared decisionmaking regarding potential benefits and harms of surgery. Furthermore, perioperative risk assessment tools fail to include dialysis treatment as a risk factor, potentially leading to the use of unvalidated risk indices in this unique population.

The aims of this study were to ascertain the odds of non-fatal cardiovascular and infectious postoperative outcomes in patients receiving dialysis compared to patients with normal kidney function. A secondary aim was to explore the effects of age and non-kidney comorbidity on excess odds for these outcomes.

\section{Methods}

This systematic review adhered to the Preferred Reporting Items for Systematic Reviews and Meta-analysis (PRISMA) [7] and Meta-analysis Of Observational Studies in Epidemiology (MOOSE) [8] checklists, with a protocol registered in PROSPERO (CRD42017076565). This paper focuses on the pre-specified secondary outcomes of the registered protocol pertaining to morbidity after elective surgery in patients on chronic dialysis. The search strategy and statistical analysis were adopted from this registered protocol [4].

\section{Search strategy}

Without language restriction, MEDLINE, Embase and Cochrane Controlled Register of trials (CENTRAL) were searched for studies published until January 2020, using a combination of relevant keywords including surgery, dialysis, postoperative, perioperative, mortality, morbidity and their variants (Details of strategy in Supplementary Table 1, 2, 3, 4). Exploded MeSH terms for perioperative medicine and chronic dialysis patients were also used. Search terms were modified to correspond to the tree structure and descriptors of the two databases. Further studies were sought by manually searching reference lists of the relevant articles. In addition, tangential electronic exploration using links to related texts was also performed. Case-control studies, animal studies, opinion papers, case reports and editorials were excluded.

No existing reviews were identified in the Cochrane Database of Systematic Reviews (CDSR), Database of Abstracts of Reviews of Effects (DARE), NIHR Health Technology Assessment (NIHR HTA) programme and the National Institute for Health and Clinical Excellence (NICE) websites.

\section{Selection criteria}

All cohort studies that measured and reported postoperative morbidity in adult (aged 18 years or older) chronic dialysis patients concurrently with patients who had normal kidney function were considered for inclusion. Normal kidney function was defined as a serum creatinine of less than $110 \mu \mathrm{mol} / \mathrm{l}$ or the absence of International Classification of Disease (ICD) coding of chronic kidney disease. All surgical disciplines were considered, including general, orthopaedic, cardiac, vascular and urology/ gynaecological surgery. Studies where the proportion of urgent or emergent surgeries was less than $20 \%$ were included. Studies involving kidney transplantation, dialysis access surgery, and both endovascular and endoscopic procedures, were not included. Patients requiring chronic renal replacement therapy (CRRT) for an acute kidney injury undergoing surgery were not eligible for inclusion.

\section{Data extraction and outcome definition}

Two researchers (DP and AN) independently screened all abstracts identified in the initial search to assess conformity with selection criteria. Disagreements were resolved by a third reviewer (MF). Data on the following characteristics were extracted independently by two investigators using a standard electronic data extraction form: type of surgery, numbers of dialysis patients, number of patients with normal kidney function, location (defined as Asia-Pacific, Europe and North America), summary statistics for patient baseline characteristics (including cardiovascular disease, peripheral vascular disease, diabetes mellitus, hypertension and smoking status), frequency of postoperative outcomes, and adjusted odds ratios where available.

The primary outcomes were postoperative myocardial infarction, stroke, surgical site infections (both superficial and deep), sepsis and pneumonia, defined as either within 30-days or within the same hospitalisation as the index surgery. Secondary outcomes were postoperative packed red cell transfusion, thromboembolic events and unplanned return to theatre. The definition used by a given study for each complication, if present, was noted 
at the time of data extraction. Furthermore, severity of complications, measured using the Clavien-Dindo Classification were recorded if available. The Clavien-Dindo classification is a scale from 1 to 5 measuring the implications of a post-operative complication on a patient's treatment course and outcome [9]. Grade 1 refers to any deviation to the usual postoperative course, grade 2 refers to the need for complication-specific pharmacotherapy, grade 3 refers to the need for endoscopic or radiological intervention, grade 4 refers to the need for intensive care admission, and grade 5 refers to death arising from the post-operative complication.

Two independent reviewers assessed the methodological quality of each study using the Newcastle-Ottawa Scale (NOS), which employs a star system to evaluate the selection of the study groups (0-4 stars), comparability of the groups (0-2 stars), and ascertainment of the outcome of interest (0-3 stars) [10]. The GRADE (grading of recommendations assessment, development, and evaluation) approach was used to assess the certainty of evidence for each outcome [11]. The certainty of evidence was classified into one of four categories; high, moderate, low and very low (Supplementary Table 6).

\section{Statistical analysis}

For all outcomes, an unadjusted odds ratio [OR] and 95\% CI were calculated using the number of events in each group. Summary estimates were calculated using inverse variance weighted random effects meta-analysis [4]. Both individual study and summary odds estimates were displayed in forest plots by each surgical type. Surgical types were not combined in any analyses owing to clinical heterogeneity. Adjusted OR and 95\% CI were recorded if studies performed a multivariable analysis, adjusting for age as a minimum.

To assess the relationship between the unadjusted effect size and important study level covariates, meta-regression was performed using the random effects model with two categories of predictor variables: study characteristics, including study quality (as per NOS), single versus multicentre cohorts, continents, study duration, and single procedure studies versus composite procedures, and patient characteristics, including age and relative prevalence of diabetes mellitus or ischemic heart disease among dialysis patients compared to non-dialysis patients, including age and pre-operative co-morbidity burden (ischemic heart disease and diabetes) [4].

L'Abbé plots were generated to identify studies responsible for divergent results [12]. Inter-rater reliability of study selection was assessed using Cohen's kappa. A funnel plot and Egger's test for funnel plot asymmetry were used to assess publication bias. Heterogeneity was assessed using $I^{2}$ [13].
Statistical analysis was performed with Stata 14.0 for Windows. Statistical significance was defined as a twosided $p$-value $<0.05$.

\section{Results \\ Study selection and characteristics}

In total, 5,135 abstracts were reviewed, from which 115 full-text articles were retrieved and evaluated (Fig. 1). Forty-nine studies, involving 10,513,934 patients with normal kidney function and 43,092 chronic dialysis patients, satisfied the inclusion criteria (Table 1: Summary of baseline characteristics of studies included). The definition of chronic dialysis varied across studies, with 22 studies using registry-based definitions, six using International Classification of Disease Coding (ICD) and the remaining studies confirming chronic dialysis status by medical chart reviews. Non-emergent cardiac surgery was the most commonly reported type of surgery (28\%) [14-27], followed by general surgery (24\%) [2, 28-38], orthopaedic surgery (22\%) [39-49], vascular surgery (16\%) [50-56], and urologic/gynaecologic surgery (8\%) [57-60]. Twenty-six of the 49 studies assessed a single surgical procedure $[18-20,22,24-26,32,34,36-39$, $41-43,45,48-50,53,54,56-59]$, while the remaining 23 studies examined a mixture of discipline-specific surgical interventions.

Thirty-seven studies failed to indicate dialysis modality, ten studies specifically examined haemodialysis patients only [18, 20, 22, 23, 26, 31, 33, 39, 43, 50, 57], and two studies looked at peritoneal dialysis patients separately [32, 49].

Of the 49 studies, 19 reported findings from a single centre $[14,16,18-23,25,32,35,41,43,47,50,51,57$, $59,61]$ and only three collected data prospectively [14, $18,43]$. Twenty-three studies extracted information from existing data registries while the remaining extracted information from re-examined health records. Thirty-three studies were reported from North America [2, 15-17, 21-24, 27-29, 31, 33-37, 40, 42, 44-46, 49, 51-56, 58, $60,62]$, ten from Asia [18-20, 26, 30, 38, 39, 43, 47, 48, $50,59]$ and four from Europe [14, 32, 41, 57]. Thirtyfour studies were published after $2010[2,14,16,22-24$, $27-31,33,34,36-50,53-56,58-60,62]$.

All 49 studies reported age and gender, but comorbidities were less consistently described, with 37 (76\%) studies reporting the prevalence of diabetes mellitus [2, 14$19,21,23-30,33,34,36-41,43,46,47,49-54,60$, 62], 32 (65\%) reporting ischemic heart disease (IHD) [2, 14$21,23,25,27-30,34,36-41,46-49,51-54,60,62], 20$ (41\%) reporting smoking status $[17,19,23,25,26,28$, $29,33-35,37,38,46,49-52,54,60-62]$, and $14(29 \%)$ studies reporting all three comorbidities $[19,25,28,29$, $33,37,46,49,51,52,54,60,63]$. 

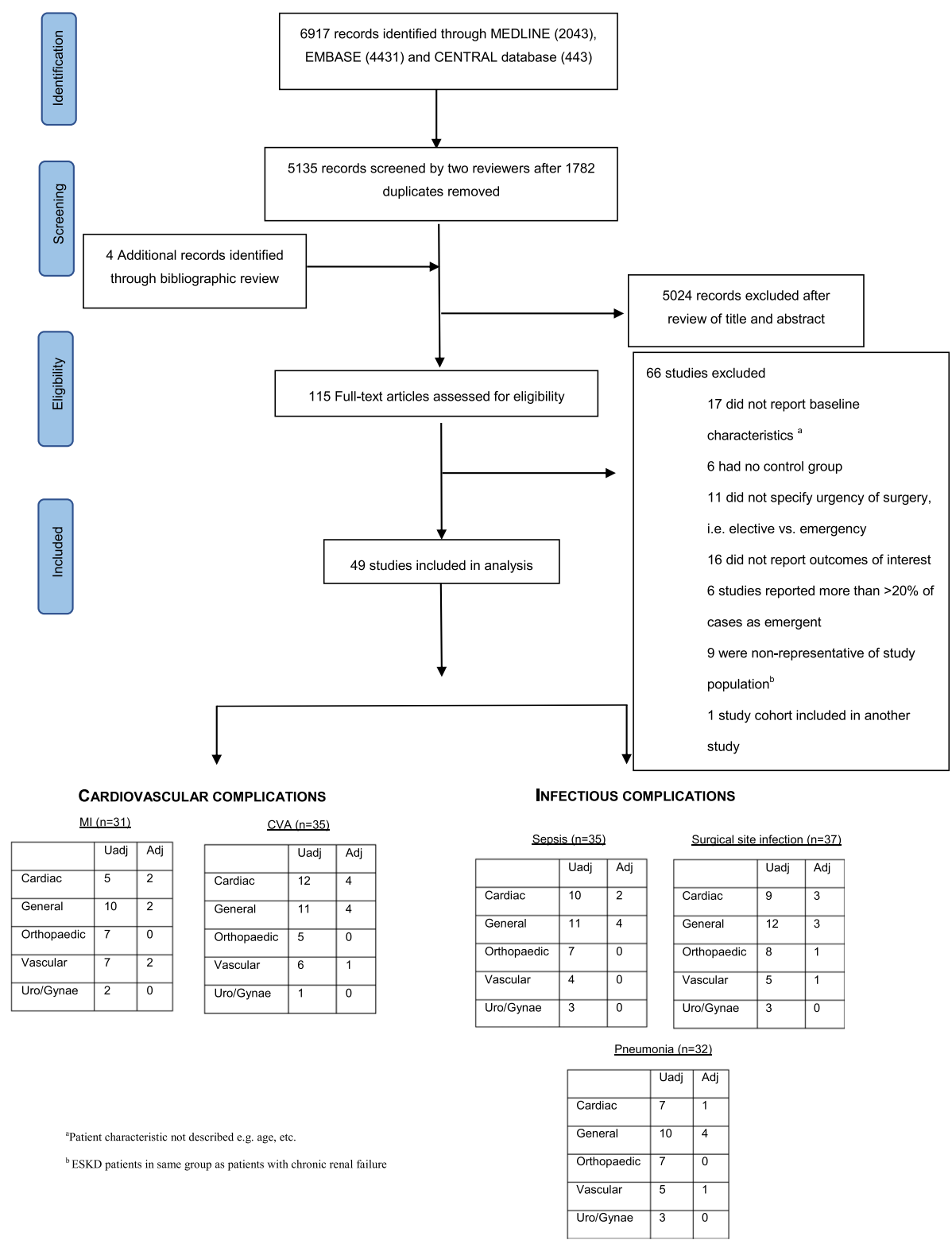

Fig. 1 PRISMA flow diagram of study selection

\section{Cardiovascular complications}

Myocardial infarction Myocardial infarction was reported in 31 studies involving 25,775 patients receiving chronic dialysis and 4,008,163 patients with normal kidney function. Studies in general surgery most frequently reported myocardial infarction as a complication (10 of 12 studies). The incidence of postoperative myocardial infarction across all individual studies ranged from 0 to $6.7 \%$ in dialysis patients and $0-4.0 \%$ in patients with normal kidney function. The unadjusted odds of myocardial infarction was higher for chronic dialysis patients than for patients with normal kidney function in all surgical specialities, with the lower bounds of the
95\% CI of the relative risk estimates for each surgical discipline equal to or greater than 1.0 (Fig. 2a). The highest odds was following orthopaedic surgery (7 studies, 3902 dialysis patients, OR 4.13, 95\% CI 2.24-7.61, $\mathrm{I}^{2}=76.4 \%, p<0.001$, moderate certainty evidence). Adjusted odds ratio estimates, with age as a minimum covariate, was reported by 5 studies in which the excess odds was attenuated but remained high following general surgery (2 studies, 10,443 dialysis patients, OR $1.8395 \% 1.29-2.36, \mathrm{I}^{2}=0.0, P=0.852$, low certainty evidence).

Stroke Thirty-five studies reported postoperative stroke, involving 34,400 chronic dialysis patients and 4,286,805 


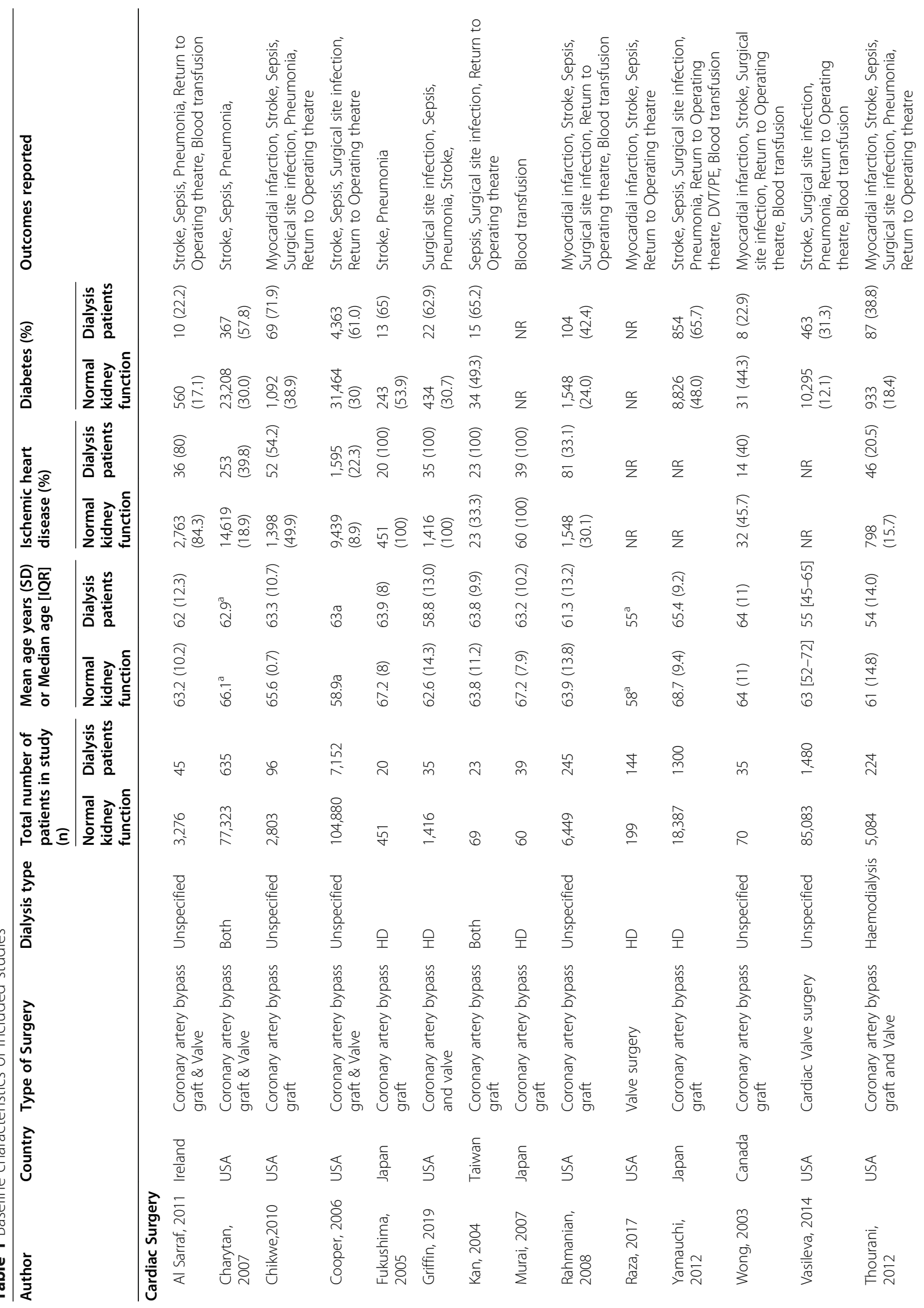




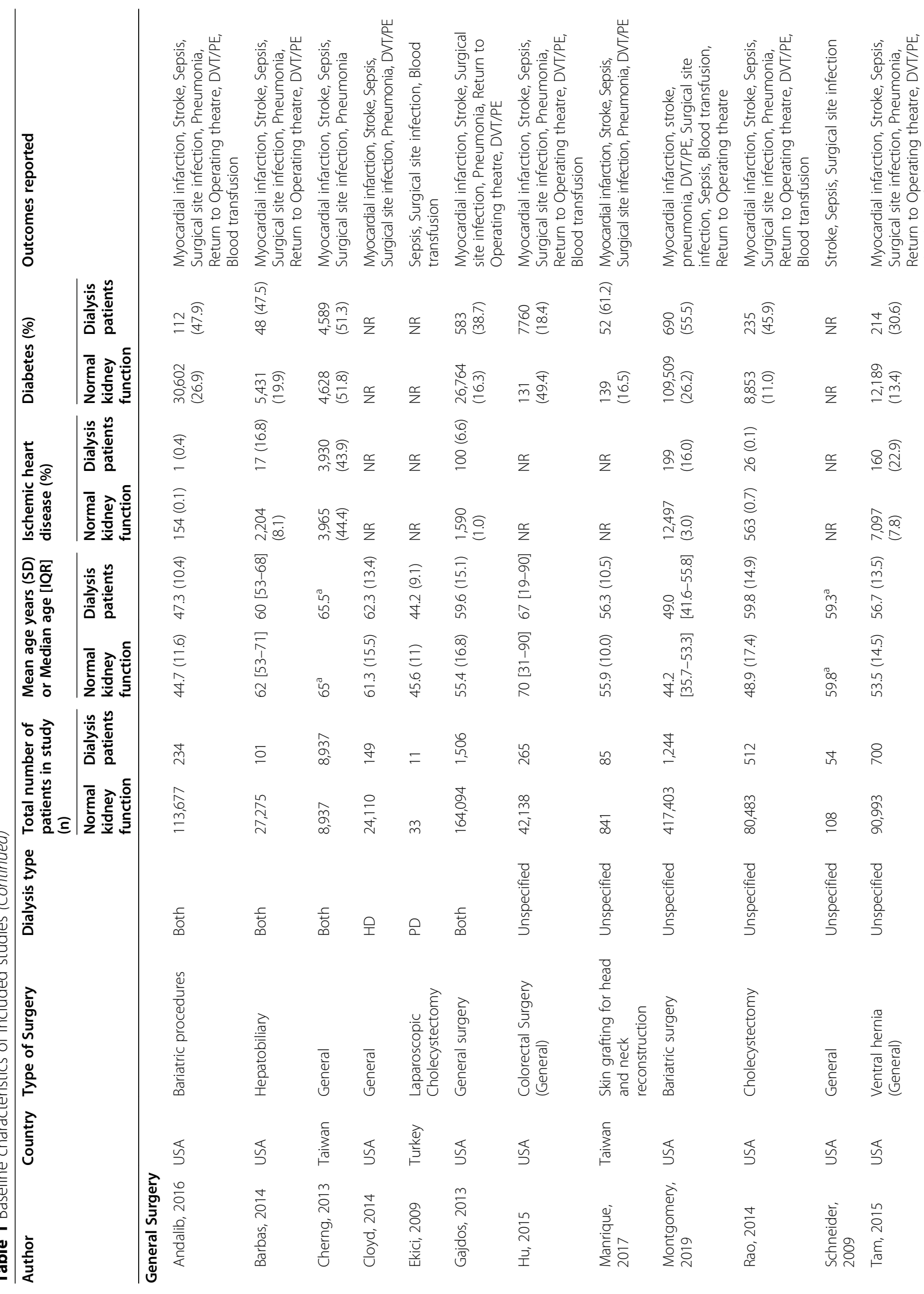




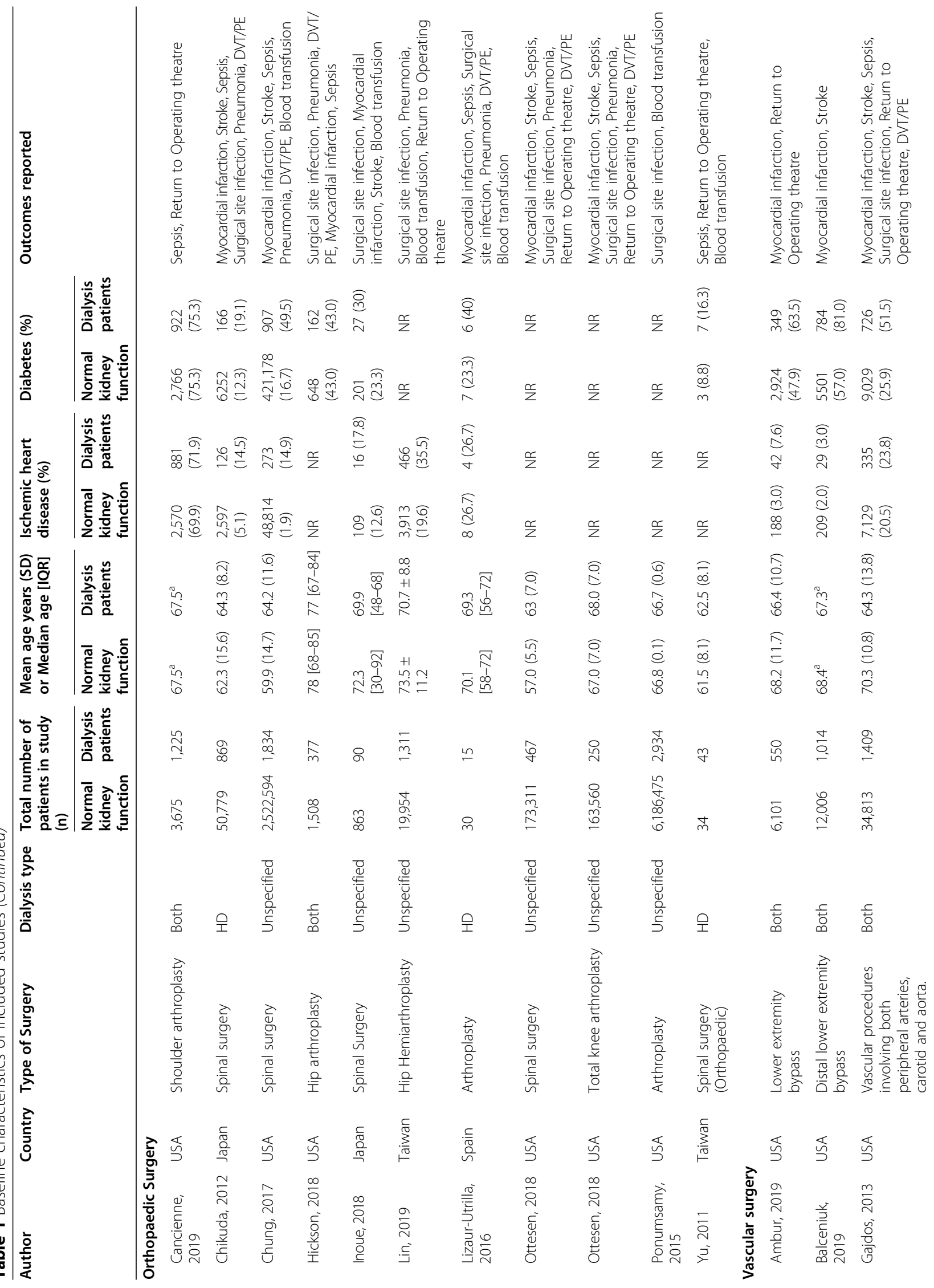




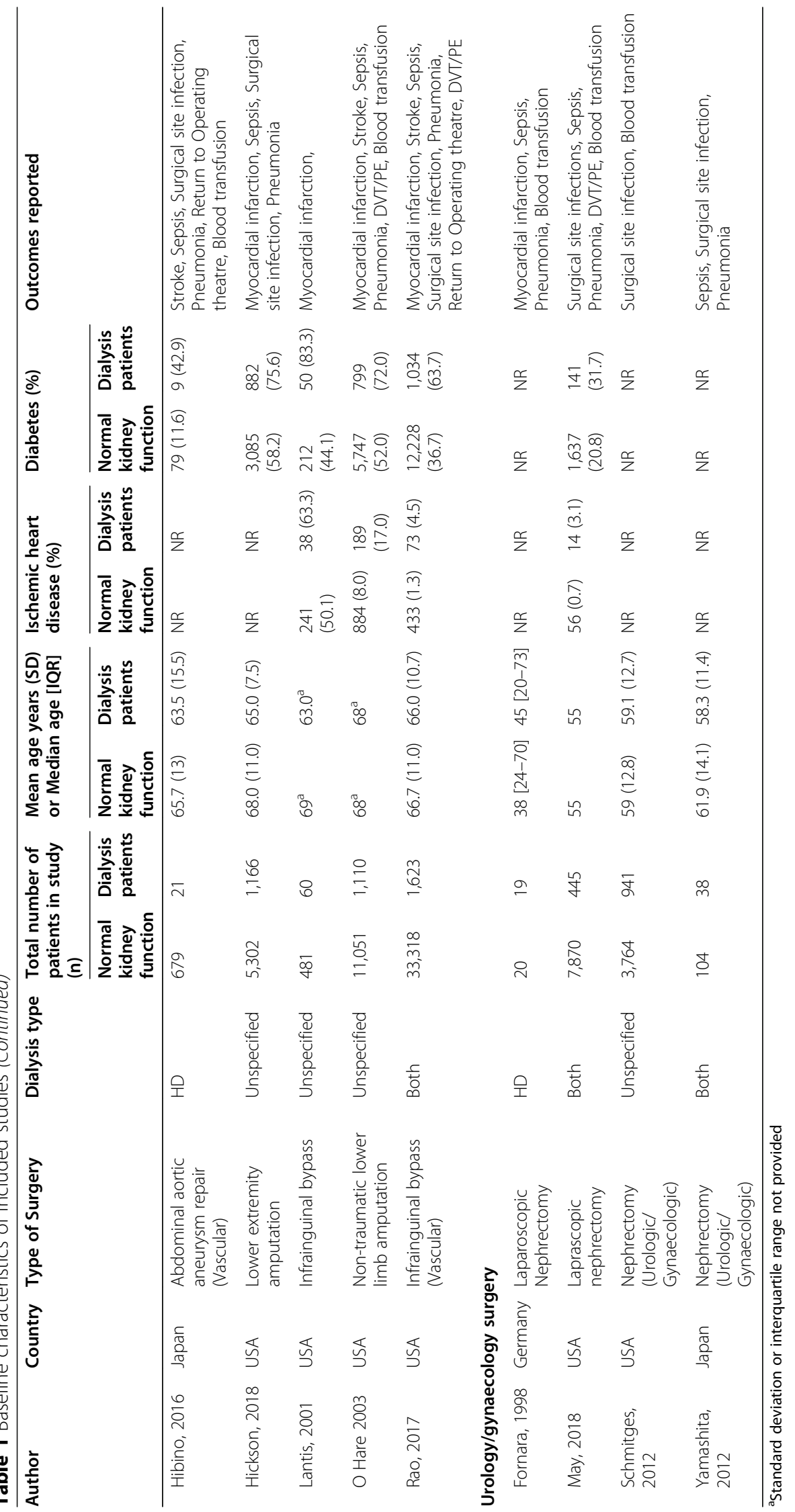


A) Postoperative myocardial infarction odds for patients on chronic dialysis

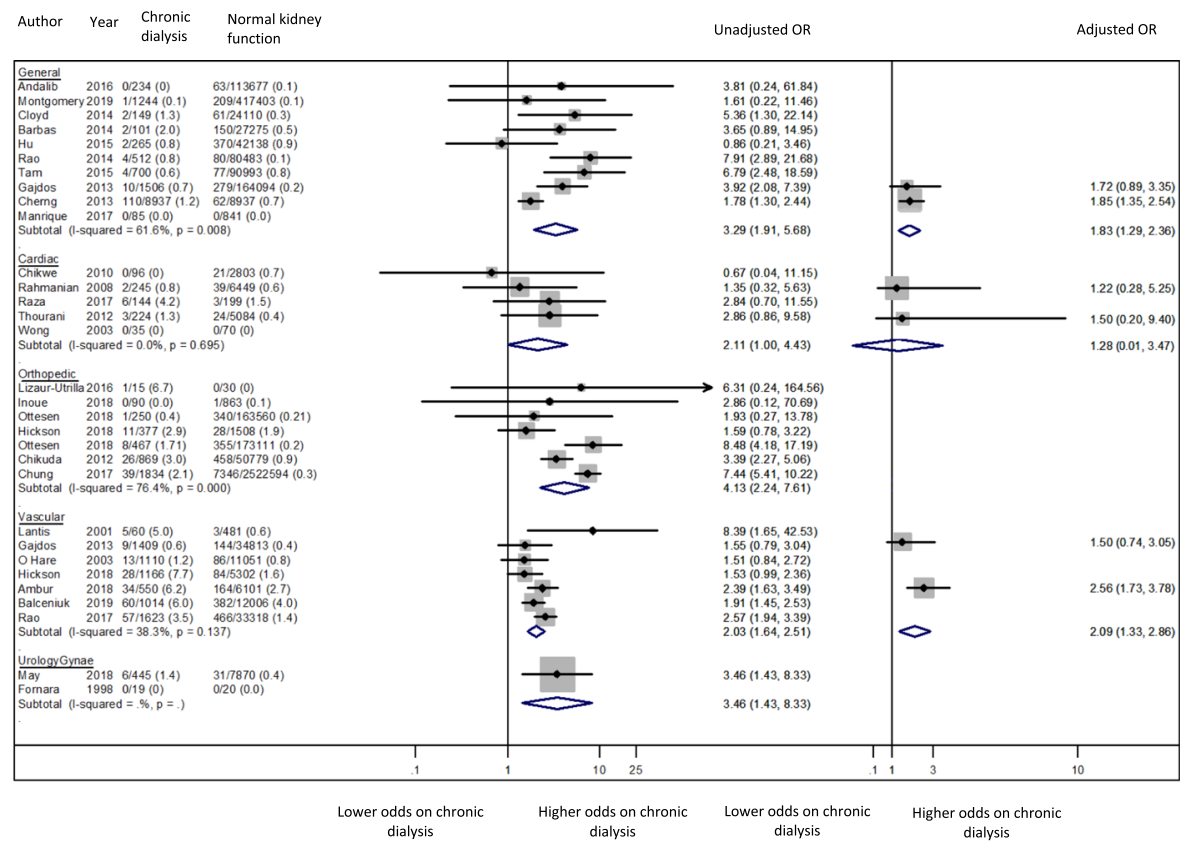

B) Postoperative stroke odds for patients on chronic dialysis

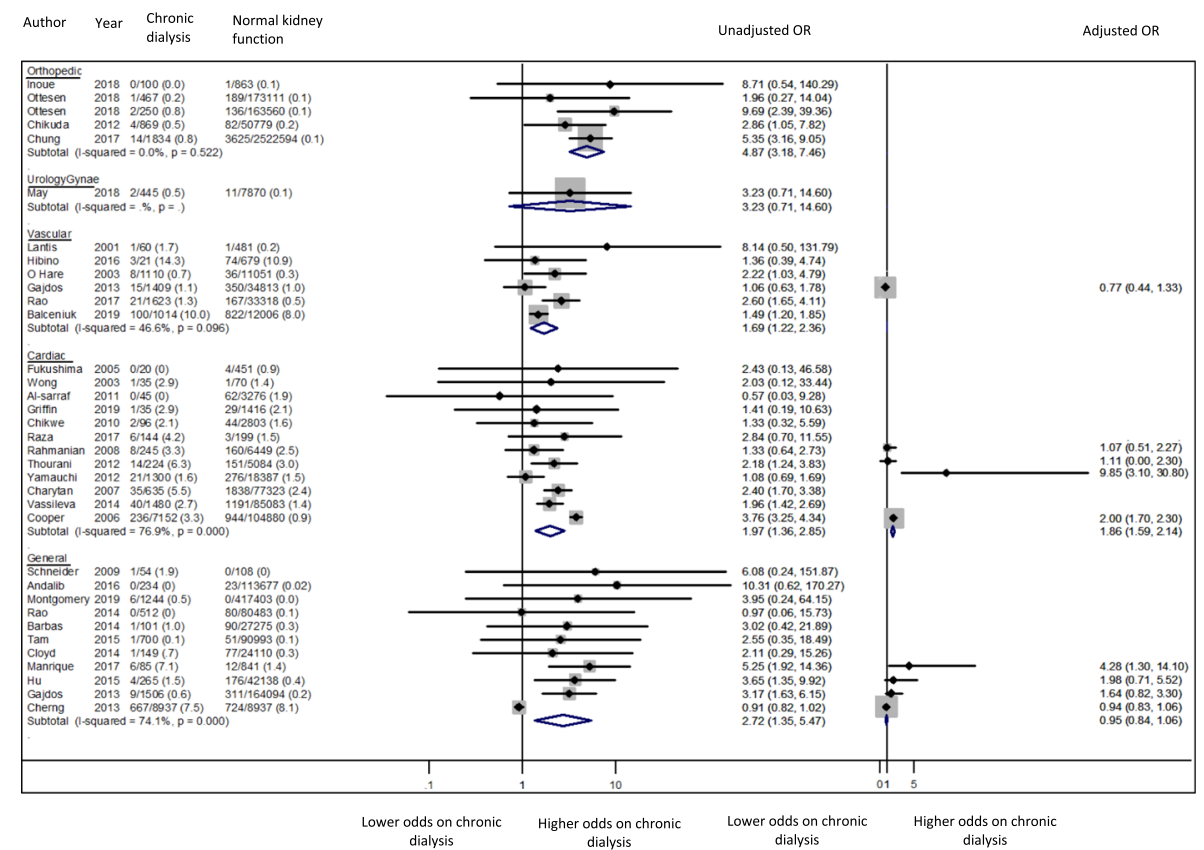

Fig. 2 Cardiovascular Complications. a Postoperative myocardial infarction odds for patients on chronic dialysis. b Postoperative stroke odds for patients on chronic dialysis

patients with normal kidney function. Stroke was most frequently reported by studies in cardiac surgery (12 of 14 studies), followed by studies in general surgery (11 of 12 studies). The incidence of stroke across all thirty individual studies ranged from 0 to $14.3 \%$ in chronic dialysis patients, and $0-10.9 \%$ in patients with normal kidney function. The median reported incidence of stroke in chronic dialysis patients by sub-specialty was highest following cardiac surgery at $2.9 \%$, compared to $1.5 \%$ in their non-dialysis counterparts. Studies did not 
differentiate between off-pump coronary artery bypass surgery and conventional coronary artery bypass. Only one study involving urologic or gynaecologic surgery reported the incidence of stroke. The unadjusted odds ratio of stroke was considerably higher for patients receiving chronic dialysis compared to patients with normal kidney function in all surgical disciplines (Fig. 2b). The highest odds observed was following orthopaedic surgery (5 studies, 3,520 dialysis patients, OR 4.87, 95\% CI $3.18-7.46, \mathrm{I}^{2}=0.0 \%$, p for heterogeneity 0.52 , moderate certainty evidence). Adjusted odds ratio estimates were provided by 9 studies. The summary odds risk estimate remained elevated following cardiac surgery (4 studies, 8921 dialysis patients, OR 1.86, 95\%CI 1.59$2.14, \mathrm{I}^{2}=56.2 \%, p=0.08$, low certainty evidence) but was not significantly different from that of patients with normal kidney function following general surgery (4 studies, 10,793 dialysis patients, OR 0.95 , 95\%CI $0.84-$ $1.06, \mathrm{I}^{2}=0.00, p=0.40$, low certainty evidence).

\section{Infectious complications}

Sepsis Sepsis was reported in 35 studies, involving 3, 996,044 patients with normal kidney function and 30, 468 dialysis patients, and was most frequently reported following general surgery (11 of 12 studies). Across all studies, the incidences of sepsis ranged from 0 to $21.8 \%$ in dialysis-dependent patients and $0-11.8 \%$ in patients with normal kidney function. When comparing incidence rates between specialities, the highest median reported rate of sepsis in dialysis patients was 10.0\% after general surgery. Meta-analysis showed that being on chronic dialysis was associated with an increased odds of developing postoperative sepsis, irrespective of surgical discipline (Fig. 3a). The highest excess odds was seen following orthopaedic surgery (7 studies, 3,855 dialysis patients, OR $5.41,95 \%$ CI $2.88-10.16, \mathrm{I}^{2}=87.5 \%$ $\mathrm{p}$ for heterogeneity $<0.001$, moderate certainty evidence). Summary odds ratio estimates from the adjusted results attenuated the excess odds (2 cardiac surgery studies, 1,545 dialysis patients, OR $2.77,95 \%$ CI $1.47-$ $4.07, \mathrm{I}^{2}=7.7 \%, p=0.30$, low certainty evidence; 4 general surgery studies, 9388 dialysis patients, OR $2.42,95 \%$ CI $2.12-2.72, \mathrm{I}^{2}=85.3 \% p<0.001$, low certainty evidence).

Surgical site infection Thirty-seven studies involving 7 , 877,144 patients with normal kidney function and 36, 414 patients receiving chronic dialysis reported surgical site infections. The incidences of surgical site infections across the all studies ranged from 0 to $43.5 \%$ in dialysis patients and $0-20.3 \%$ in patients with normal kidney function. Compared to other specialties, the highest median incidence of surgical site infections for chronic dialysis patients was $7.2 \%$ following general surgery. The odds ratio of surgical site infection was higher among patients receiving chronic dialysis compared to patients with normal kidney function after all types of surgery except following urologic and gynaecologic procedures (Fig. 3b). There was almost a 3-fold increased excess odds of surgical site infections following cardiac surgery (9 studies, 10,590 dialysis patients, OR 2.86, 95\% CI $1.98-4.14, \mathrm{I}^{2}=68.7 \% \mathrm{p}$ for heterogeneity $<0.001$, moderate certainty evidence). Meta-analysis of adjusted odds ratios for cardiac surgery attenuated the heightened odds in dialysis patients (3 cardiac surgery studies, 8,697 dialysis patients, OR $1.99,95 \%$ CI $1.52-2.45, \mathrm{I}^{2}=79.6$, $p=0.007$, low certainty evidence).

Pneumonia Thirty-two studies involving $4,185,864$ patients with normal kidney function and 28,426 dialysisdependent patients reported postoperative pneumonia. Ten of the 12 studies in general surgery reported pneumonia as a complication. The incidences of pneumonia across all studies ranged from 0 to $52.4 \%$ in dialysis patients and $0-13.7 \%$ in patients with normal kidney function. The postoperative odds ratio of developing postoperative pneumonia was higher in chronic dialysis patients compared to patients with normal kidney function across all surgical disciplines (Supplementary Figure 1). Adjusted odds ratios were provided by 6 studies. The excess odds remained elevated following general surgery (4 general surgery studies, 10,793 chronic dialysis patients, OR 1.54, 95\% CI 1.37-1.71, $\mathrm{I}^{2}=51.9 p=0.10$, moderate certainty evidence).

Other surgical outcomes Patients receiving chronic dialysis were also at higher odds of other non-fatal surgical outcomes compared to patients with normal kidney function, including unplanned return to theatre (highest following general surgery; 7 studies 4,562 dialysis patients, OR 2.75, 95\%CI 1.98-3.81, $\mathrm{I}^{2}=95.2 \%, p=0.001$, low certainty evidence), blood transfusion requirement (highest following cardiac surgery; 6 studies, 3,144 patients, OR 4.23, 95\%CI 2.80-6.37, $\mathrm{I}^{2}=86 \%, p=0.001$, low certainty evidence), and venous thromboembolism (highest following general surgery; 9 studies, 4,796 patients, OR 1.75, 95\%CI 1.25-2.45, $\mathrm{I}^{2}=20.6 \%, p=0.012$, low certainty evidence). (See Supplementary Table 5).

\section{Meta-regression}

A series of weighted univariable random-effects metaregression analyses were performed to examine the relationship between unadjusted odds ratios and characteristics that may explain their variation. No study characteristic, including median year of study recruitment, study continent, cardiac versus non-cardiac surgery and overall study quality as assessed by the Newcastle Ottawa Scale (Supplementary Table 4), 
A) Postoperative sepsis oddsfor patients on chronic dialysis

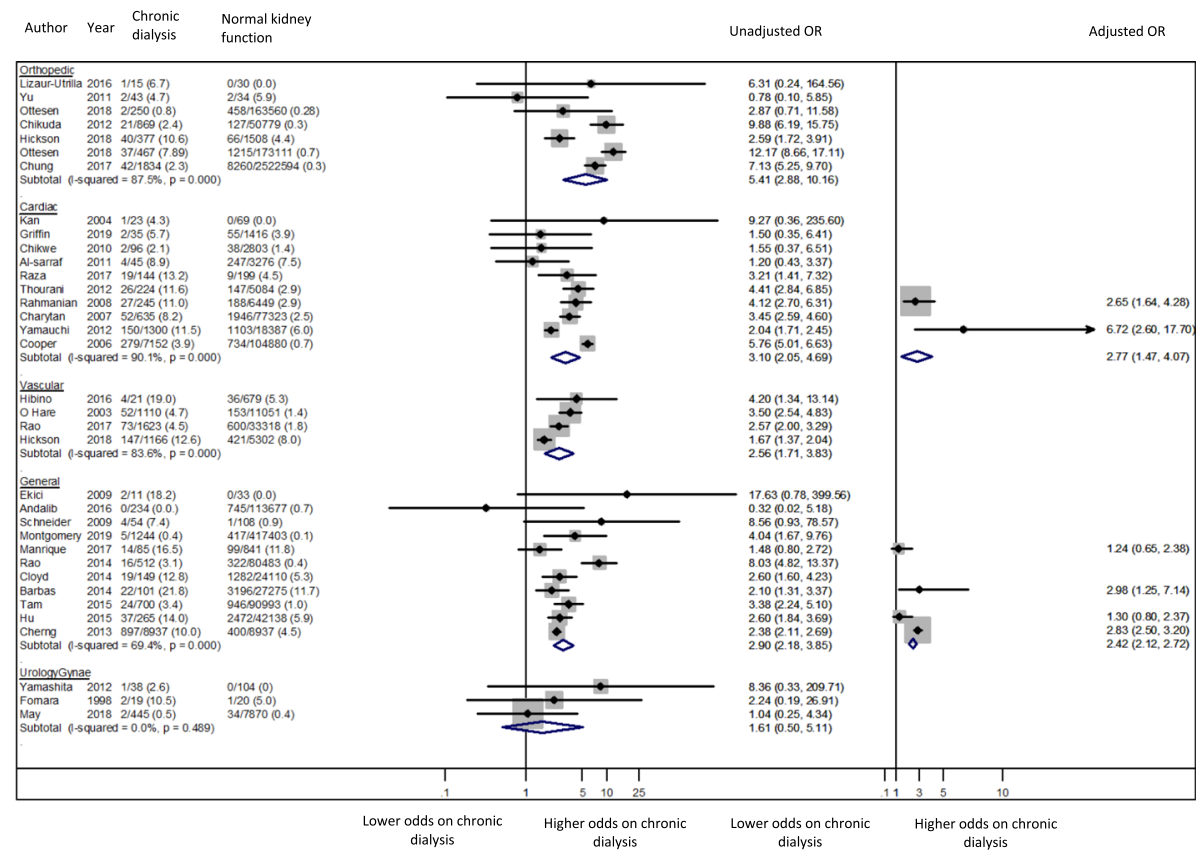

B) Postoperative surgical site infection odds for patients on chronic dialysis

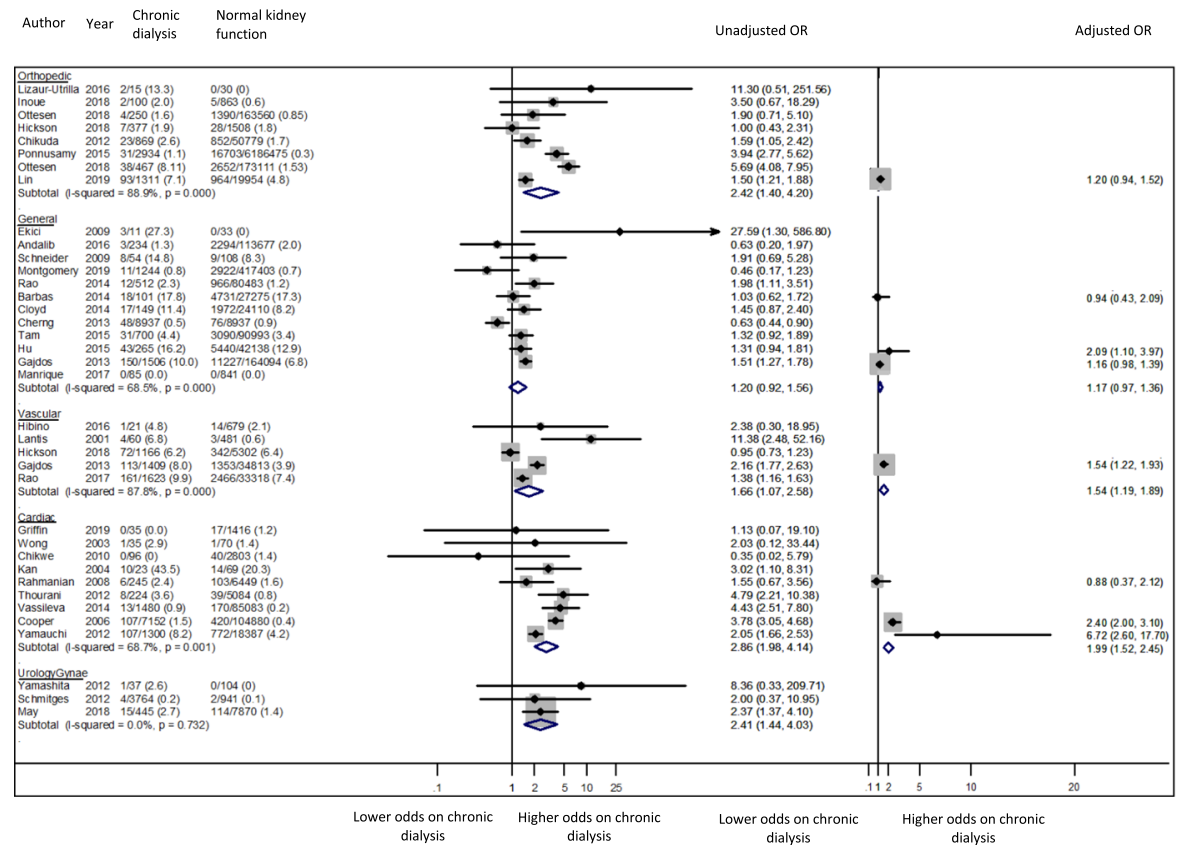

Fig. 3 Postoperative Infectious Complications. a Postoperative sepsis odds for patients on chronic dialysis. b Postoperative surgical site infection odds for patients on chronic dialysis

explained the observed heterogeneity in odds ratio for any of the non-fatal outcomes. However, several patient characteristics were predictive (Supplementary Figure 2A-2F). Firstly, meta-regression of the weighted mean age of each study on postoperative myocardial infarction and stroke demonstrated inverse linear relationships for both outcomes: slope $-0.05,95 \%$ CI $(-0.09--0.02) p<$ 0.001 for myocardial infarction, and slope $-0.04,95 \% \mathrm{CI}$ $(-0.08-0.01) p=0.031$ for stroke. A similar inverse linear relationship was observed between the excess risk of myocardial infarction and prevalence of diabetes (slope $-0.02,95 \%$ CI $-0.03--0.01, p=0.004$ ), and also 
between stroke risk and prevalence of ischemic heart disease (slope $-0.02,95 \%$ CI $-0.04--0.01, p=0.001$ ); the latter relationship was maintained in multivariable metaregression adjusted for age and diabetes mellitus (slope - 0.02, 95\%CI -0.01- $-0.01, p=0.006$ ).

An inverse linear relationship was also seen with the excess odds of pneumonia and age (slope $-0.04,95 \% \mathrm{CI}$ $-0.06--0.01, p=0.003)$ and prevalence of diabetes (slope - 02, 95\%CI $-0.02-0.0, \mathrm{p}=0.003$ ).

Univariable meta-regression identified a linear relationship between the risk of surgical site infections and prevalence of ischemic heart disease (slope $0.03,95 \% \mathrm{CI}$ $0.01-0.05, \mathrm{p}=0.006$ ).

\section{Risk of Bias}

Morbidity was inconsistently reported across studies: surgical site infection (37 studies) and sepsis (35 studies) were the most frequently reported. Seventeen of the 49 studies did not have explicit definitions of complications. Two studies [29, 60] reported post-operative complications graded by the Clavien-Dindo classification of surgical complications. Outcomes that were reported were of good quality, but comparability of patient groups on the basis of analysis was poor in $24(49 \%)$ studies due to the absence of multivariable adjustment for patient demographics and co-morbidities [18-20, 22, 25-28, 32, 34, 35, 37-39, 41, 43, 46, 47, 49-51, 57, 59-61]. (Supplementary Table 4: Methodological quality of each study assessed by the NOS scale). Neither the funnel plot (Supplementary Figures 3A-E) nor Egger's test ( $p=$ $0.328)$ suggested evidence of publication bias. Inter-rater variability between the two independent reviewers was strong $(\kappa=0.81)$.

The certainty in the quality of evidence was deemed to be low. The quality of evidence was downgraded both due to concerns with risk of bias (vide-supra), and for inconsistency due to residual heterogeneity. Having said that, the large magnitude of the odds estimate improved the strength of the evidence (Table S6).

\section{Discussion}

This study demonstrated that patients with ESKD requiring chronic dialysis have increased odds of postoperative cardiovascular complications (myocardial infarction and stroke) and infectious complications (sepsis, surgical site infections and pneumonia) compared to patients with normal kidney function. This meta-analysis demonstrated a two- to fivefold increase in odds of postoperative myocardial infarction and stroke for patients on chronic dialysis, irrespective of surgical discipline. ESKD and dialysis are well established independent risk factors for adverse cardiovascular events; this relationship can be attributed to a number of non-traditional risk factors including inflammation, anaemia, calcium-phosphate imbalance and oxidative stress, in addition to dialysis specific factors such as intradialytic hypotension and myocardial stunning [64]. However, in studies that reported multivariable adjusted odds ratios, this excess risk was substantially attenuated. For instance, the odds of a postoperative stroke for chronic dialysis patient following general surgery was no different when compared to a patient with normal kidney function. Furthermore, meta-regression demonstrated that the excess odds of myocardial infarction attributable to receiving chronic dialysis treatment was less apparent with older age and in the presence of diabetes mellitus. A similar interaction was also observed between stroke risk, older age and presence of ischaemic heart disease. Taken together, these findings suggest that age and non-dialysis related comorbidities, including diabetes and ischemic heart disease, contribute significantly to the heightened odds observed, and dialysis while a risk factor for adverse outcomes is not itself the dominant driver of perioperative morbidity in this population. Currently, patients wait-listed for kidney transplantation undergo screening for coronary artery disease using non-invasive functional testing to identify occult disease and consider revascularization [64]. However, no such recommendations exist for patients on chronic dialysis considering elective surgery.

The odds of sepsis, surgical site infections and pneumonia, were also consistently elevated in patients on chronic dialysis compared to patients with normal kidney function across all surgical disciplines, with the exception of surgical site infections in general surgery and postoperative sepsis following urology/gyane surgery. The excess odds of sepsis among chronic dialysis patients remained elevated, even after adjusting for age and comorbidities, with the odds being more than twofold higher after cardiac and general surgery. The magnitude of the odds of pneumonia and surgical site infections for chronic dialysis patients was substantially reduced in adjusted analyses. Interestingly, metaregression showed neither patient age nor the presence of diabetes mellitus appeared to modify the excess odds of sepsis or surgical site infections attributable to being on dialysis. These results differed to those for myocardial infarction and stroke, suggesting that the observed excess risk of infectious complications may be explained by the impaired immunity associated with ESKD and chronic dialysis [65]. The use of immunosuppression was reported in very few studies, such that determining their influence was not possible even though this is an important clinical consideration in patients with ESKD. Another potential reason for the elevated odds ratios seen in chronic dialysis patients may be related to definition of ESKD used in the various studies. A large number of studies were undertaken using data from 
registries such as the American College of Surgeons National Surgical Quality Improvement Program (ACS NSQIP), Vascular Quality Improvement Program and The Society of Thoracic Surgeons Database (STS), where dialysis dependency was defined as a patients requiring any form of dialysis within 2 weeks of surgery. Thereby potentially including patients with dialysis-dependent acute kidney injury in the study cohort. Patients with acute kidney injury carry substantially increased mortality and morbidity risk and therefore may have exaggerated the findings [66]. Studies also poorly reported dialysis treatment-related variables known to predispose chronic dialysis patients to infections, such as the presence of indwelling medical devices (central venous catheter, arteriovenous grafts and peritoneal dialysis catheters which provide an entry point for organisms) [67]. Furthermore, other important dialysis factors known to influence mortality and morbidity among dialysis patients in general, including dialysis vintage, dialysis modality and adequacy, were insufficiently reported $[68,69]$. Therefore, the competing influences of these factors were not able to be evaluated. Indeed, more advanced disease or delayed surgery may also explain the observed differences. Data on other important surgical technique factors such as operational time and anesthesia type were also not available for analysis. It is also possible that variation between studies in the outcomes reported and definitions contributed to the differences in observed odds ratios.

In a prior meta-analysis, a sub-group analysis of 13 cohort studies involving 97,709 patients with normal kidney function and 27,501 with non-dialysis-requiring chronic kidney disease identified kidney dysfunction as an important, independent risk factor for composite postoperative cardiovascular events (arrhythmia, heart failure, angina, cardiac arrest, pulmonary oedema) following vascular and general surgery [70]. The results of our meta-analysis support these findings and extend them by demonstrating that the elevated postoperative odds of cardiovascular and infectious complications likely also apply to patients with kidney disease treated with dialysis.

\section{Limitations of analysis}

To allow for greater generalisability, a comprehensive search strategy was used to identify a large number of dialysis patients across all elective surgical types. Despite attempts to adjust for potentitally confounding variables, such as age, indication bias with residual confounding could not be excluded. Although most studies reported sufficient details for the population to be included, many of the studies did not report potentially important confounding variables, such as primary kidney disease, dialysis modality and vintage, dialysis access type, residual kidney function or use of immunosuppression. Publication bias could also not be confidently ruled out, especially for those outcomes reported by only a few studies (e.g. thromboembolic complications). In addition, there was uncertainty about how well events were captured and reported. Therefore, the true frequency of postoperative complications in dialysis patients following major elective surgery is unknown. The absence of an objective and reproducible approach to classification of complications, such as the Clavien-Dindo classification, limited fair comparison of surgical outcomes between different patient populations and surgery types [9]. Finally, the selection of study cohorts was of good quality, but comparability of patient groups on the basis of analysis was poor in $24(49 \%)$ studies due to the absence of multivariable adjustment for patient demographics and comorbidities.

Future studies need to be more thorough in reporting patient baseline dialysis characteristics, procedural information and postoperative morbidity to allow for more informative analyses with adjustment for confounding. For instance, the microbiology of pathological organisms was not reported by any studies. Recovery of organisms from cultured specimens may not only inform future guidelines for empiric therapy in the perioperative setting, but also indicate potential sources. This is of particular importance given the higher odds of infectious complications demonstrated in this review [65]. Moreover, current perioperative risk assessments tools are unvalidated in chronic dialysis patients and fail to incorporate important dialysis-related characteristics that may potentially influence perioperative outcomes [71]. Research is needed to further risk stratify patients and facilitate intervention to mitigate perioperative cardiovascular and infectious complications.

\section{Abbreviations \\ Cl: Confidence intervals; ESKD: End-stage kidney disease; GRADE: Grading of Recommendations, Assessment, Development and Evaluations; \\ ICD: International classification of disease; NOS: Newcastle-Ottawa Scale; OR: Odds Ratio}

\section{Supplementary Information}

The online version contains supplementary material available at https://doi. org/10.1186/s12882-021-02279-0.

Additional file 1: Table S1. Search strategy to be used in EMBASE. Table S2. Search Strategy for MEDLINE. Table S3. Search strategy for CENTRAL. Table S4. Methodological quality of included studies (based on Newcastle-Ottawa scale). Table S5. Meta-analysis of non-fatal postoperative complications. Table S6. Post-operative morbidity outcomes and grading of the certainty of evidence using GRADE. Figure 1: Odds of postoperative pneumonia for chronic dialysis patients. Figure 2a-g: Metaregression. 


\section{Authors' contributions}

$D P, A N, D J, E P, C H$ and MF contributed to the study concept and design. DP and $A N$ undertook the literature search and extracted data. DP, EP and MF undertook statistical analysis of the data. DP, AN, DJ, EP, CH and MF reviewed the results and interpreted the data. DP drafted the manuscript with critical revision from all authors. MF was the study supervisor. The author(s) read and approved the final manuscript.

\section{Funding}

DP is a recipient of the Royal Australasian College of Physicians Jacquot Research Entry Scholarship 2019-2020. No financial sponsor was involved in the design and conduct of the study; collection, management, analysis, and interpretation of the data; and preparation, review, or approval of the manuscript.

\section{Availability of data and materials}

The dataset used and analysed during the current study are available from the corresponding author on reasonable request.

\section{Declarations}

\section{Ethics approval and consent to participate}

N/A

\section{Consent for publication}

N/A

\section{Competing interests}

DP has received speaking honoraria from the Australian Medical Forum. DJ is a current recipient of an Australian National Health and Medical Research Council Practitioner Fellowship. DJ has previously received consultancy fees, research grants, speaker's honoraria and travel sponsorships from Baxter Healthcare and Fresenius Medical Care. CH has received funding from Janssen and GlaxoSmithKline to her institution for trial steering committee roles and research grant support to her institution from Shire, Baxter, Fresenius, and Otsuka and travel sponsorship from Otsuka.

\section{Author details}

${ }^{1}$ Metro South Integrated Nephrology and Transplant Services, Logan Hospital, Armstrong Road \& Loganlea Road, Meadowbrook, Queensland 4131, Australia. ${ }^{2}$ Faculty of Medicine, University of Queensland, Armstrong Road \& Loganlea Road, St Lucia, Queensland 4072, Australia. ${ }^{3}$ School of Medicine, Griffith University, 68 University Dr, Meadowbrook, QLD 4131, Australia. ${ }^{4}$ Department of Surgery, Sunshine Coast University Hospital, Doherty St, Birtinya, Queensland 4575, Australia. ${ }^{5}$ Metro South and Integrated Nephrology and Transplant Services, Princess Alexandra Hospital, 199 Ipswich Road, Woolloongabba, Queensland 4074, Australia. ${ }^{6}$ Translational Research Institute, Brisbane, Australia. ${ }^{7}$ Centre for Health Services Research, University of Queensland, St Lucia, Queensland 4072, Australia.

Received: 14 January 2021 Accepted: 22 February 2021 Published online: 18 March 2021

\section{References}

1. Pearse RM, Moreno RP, Bauer P, Pelosi P, Metnitz P, Spies C, et al. Mortality after surgery in Europe: a 7 day cohort study. 2012;380(9847):1059-65.

2. Gajdos C, Hawn MT, Kile D, Robinson TN, Henderson WG. Risk of major nonemergent inpatient general surgical procedures in patients on longterm dialysis. JAMA Surgery. 2013;148(2):137-43.

3. Liu JY, Birkmeyer NJO, Sanders JH, Morton JR, Henriques HF, Lahey SJ, et al. Risks of morbidity and mortality in dialysis patients undergoing coronary artery bypass surgery. Circulation. 2000;102(24):2973-7.

4. Palamuthusingam D, Nadarajah A, Pascoe EM, Craig J, Johnson DW, Hawley $\mathrm{CM}$, et al. Postoperative mortality in patients on chronic dialysis following elective surgery: a systematic review and meta-analysis. PLoS One. 2020; 15(6)::0234402.

5. Carlisle J. Pre-operative co-morbidity and postoperative survival in the elderly: beyond one lunar orbit. Anaesthesia. 2014;69(s1):17-25.

6. Tong A, Sainsbury P, Carter SM, Hall B, Harris DC, Walker RG, et al. Patients' priorities for health research: focus group study of patients with chronic kidney disease. Nephrol Dial Transplant. 2008;23(10):3206-14.
7. Moher D, Liberati A, Tetzlaff J, Altman DG. Preferred reporting items for systematic reviews and meta-analyses: the PRISMA statement. J Clin Epidemiol. 2009;62(10):1006-12.

8. Stroup DF, Berlin JA, Morton SC, Olkin I, Williamson GD, Rennie D, et al. Meta-analysis of observational studies in epidemiology: a proposal for reporting. Meta-analysis of observational studies in epidemiology (MOOSE) group. Jama. 2000;283(15):2008-12.

9. Dindo D, Demartines N, Clavien P-A. Classification of surgical complications: a new proposal with evaluation in a cohort of 6336 patients and results of a survey. Ann Surg. 2004;240(2):205-13.

10. Stang A. Critical evaluation of the Newcastle-Ottawa scale for the assessment of the quality of nonrandomized studies in meta-analyses. Eur J Epidemiol. 2010;25(9):603-5.

11. Balshem $H$, Helfand M, Schünemann HJ, Oxman AD, Kunz R, Brozek J, et al. GRADE guidelines: 3. Rating the quality of evidence. J Clin Epidemiol. 2011; 64(4):401-6.

12. Song F. Exploring heterogeneity in meta-analysis: is the L'Abbe plot useful? J Clin Epidemiol. 1999;52(8):725-30.

13. Higgins J, Thompson SG. Quantifying heterogeneity in a meta-analysis. Stat Med. 2002;21(11):1539-58.

14. Al-Sarraf N, Thalib L, Hughes A, Houlihan M, Tolan M, Young V, et al. The effect of preoperative renal dysfunction with or without dialysis on early postoperative outcome following cardiac surgery. Int J Surg. 2011;9(2):183-7.

15. Charytan DM, Kuntz RE. Risks of coronary artery bypass surgery in dialysisdependent patients--analysis of the 2001 National Inpatient Sample. Nephrol Dial Transplant. 2007;22(6):1665-71.

16. Chikwe J, Castillo JG, Rahmanian PB, Akujuo A, Adams DH, Filsoufi F. The impact of moderatetoend-stage renal failure on outcomes after coronary artery bypass graft surgery. J Cardiothorac Vasc Anesth. 2010;24(4):574-9.

17. Cooper WA, O'Brien SM, Thourani VH, Guyton RA, Bridges CR, Szczech LA, et al. Impact of renal dysfunction on outcomes of coronary artery bypass surgery: results from the Society of Thoracic Surgeons National Adult Cardiac Database. Circulation. 2006;113(8):1063-70.

18. Fukushima S, Kobayashi J, Tagusari O, Bando K, Niwaya K, Nakajima H, et al. Early results of off-pump coronary artery bypass grafting for patients on chronic renal dialysis. The Japanese Journal Of Thoracic And Cardiovascular Surgery. 2005;53(4):186-92.

19. Kan CD, Yang YJ. Coronary artery bypass grafting in patients with dialysisdependent renal failure. Tex Heart Inst J. 2004;31(3):224-30.

20. Murai N, Kodera K, Sasaki A, Asano R, Kataoka G, Ikeda M, et al. Prognosis after off-pump coronary artery bypass in patients receiving hemodialysis. Annals Of Thoracic And Cardiovascular Surgery. 2007;13(6):396-9.

21. Rahmanian PB, Adams DH, Castillo JG, Vassalotti J, Filsoufi F. Early and late outcome of cardiac surgery in dialysis-dependent patients: single-center experience with 245 consecutive patients. J Thorac Cardiovasc Surg. 2008; 135(4):915-22.

22. Raza S, Hussain ST, Rajeswaran J, Ansari A, Trezzi M, Arafat A, et al. Value of surgery for infective endocarditis in dialysis patients. Journal of Thoracic and Cardiovascular Surgery. 2017;154(1):61-70.e6.

23. Thourani VH, Sarin EL, Kilgo PD, Lattouf OM, Puskas JD, Chen EP, et al. Short- and long-term outcomes in patients undergoing valve surgery with end-stage renal failure receiving chronic hemodialysis. J Thorac Cardiovasc Surg. 2012;144(1):117-23.

24. Vassileva CM, Brennan JM, Gammie JS, Sheng S, Boley T, Saha-Chaudhuri P, et al. Mitral procedure selection in patients on dialysis: Does mitral repair influence outcomes? Journal of Thoracic and Cardiovascular Surgery. 2014; 148(1):144-50.e1.

25. Wong D, Thompson G, Buth K, Sullivan J, Ali I. Angiographic coronary diffuseness and outcomes in dialysis patients undergoing coronary artery bypass grafting surgery. European journal of cardio-thoracic surgery. 2003; 24(3):388-92.

26. Yamauchi T, Miyata H, Sakaguchi T, Miyagawa S, Yoshikawa Y, Takeda K, et al. Coronary artery bypass grafting in hemodialysis-dependent patients: analysis of Japan adult cardiovascular surgery database. Circ J. 2012;76(5):1115-20.

27. Griffin BR, Kohtz PD, Bronsert M, Reece TB, Cleveland JC, Fullerton DA, et al. Postoperative complications are not elevated in well-compensated ESRD patients undergoing cardiac surgery: end-stage renal disease cardiac surgery outcomes. J Surg Res. 2019.

28. Andalib A, Aminian A, Khorgami Z, Navaneethan SD, Schauer PR, Brethauer SA. Safety analysis of primary bariatric surgery in patients on chronic dialysis. Surg Endosc. 2016;30(6):2583-91. 
29. Barbas AS, Speicher PJ, Clary BM. Hepatic and pancreatic resection in patients with end-stage renal disease: a propensity analysis. HPB. 2014; 16(11):1016-22.

30. Cherng YG, Liao CC, Chen TH, Xiao D, Wu CH, Chen TL. Are Non-cardiac Surgeries Safe for Dialysis Patients? - A Population-Based Retrospective Cohort Study. PLoS ONE. 2013;8:3.

31. Cloyd JM, Ma Y, Morton JM, Kurella Tamura M, Poultsides GA, Visser BC. Does chronic kidney disease affect outcomes after major abdominal surgery? Results from the National Surgical Quality Improvement Program. J Gastrointestinal Surg. 2014;18(3):605-12.

32. Ekici Y, Karakayali F, Yagmurdur MC, Moray G, Karakayali H, Haberal M. Laparoscopic cholecystectomy in patients undergoing continuous ambulatory peritoneal dialysis: a case-control study. Surgical Laparoscopy, Endoscopy and Percutaneous Techniques. 2009;19(2):101-5.

33. Hu WH, Cajas-Monson LC, Eisenstein S, Parry L, Ramamoorthy S. Association of dialysis with adverse postoperative outcomes in colorectal cancer-an analysis of ACS-NSQIP. Int J Color Dis. 2015;30(11):1557-62.

34. Rao A, Polanco A, Chin E, Divino CM, Qiu S, Nguyen SQ. Safety of elective laparoscopic cholecystectomy in patients on dialysis: an analysis of the ACS NSQIP database. Surg Endosc. 2014;28(7):2208-12.

35. Schneider CR, Cobb W, Patel S, Cull D, Anna C, Roettger R. Elective surgery in patients with end stage renal disease: What's the risk? Am Surg. 2009;75(9):790-3.

36. Tam SF, Au JT, Chung PJ, Duncan A, Alfonso AE, Sugiyama G. Is it time to rethink our management of dialysis patients undergoing elective ventral hernia repair? Analysis of the ACS NSQIP database. Hernia. 2015;19(5):827-33.

37. Montgomery JR, Waits SA, Dimick JB, Telem DA. 30-day outcomes of bariatric surgery among patients with end-stage renal disease. J Am Coll Surg. 2019;229(4):S16.

38. Manrique OJ, Ciudad P, Sharaf B, Martinez-Jorge J, Moran S, Mardini S, et al. Free tissue transfers for head and neck reconstruction in patients with end-stage renal disease on Dialysis: analysis of outcomes using the Taiwan National Health Insurance Research Database. J Reconstr Microsurg. 2017;33(8):587-91.

39. Chikuda H, Yasunaga H, Horiguchi H, Takeshita K, Kawaguchi H, Matsuda S, et al. Mortality and morbidity in dialysis-dependent patients undergoing spinal surgery: analysis of a National Administrative Database in Japan. J Bone and Joint Surgery - Series A. 2012;94(5):433-8.

40. Chung AS, Campbell DH, Hustedt JW, Olmscheid N, Chutkan N. Inpatient Outcomes in Dialysis-Dependent Patients Undergoing Elective Lumbar Surgery for Degenerative Lumbar Disease. Spine. 2017.

41. Lizaur-Utrilla A, Martinez-Mendez D, Collados-Maestre I, Marco-Gómez L, Lopez-Prats FA. Elective Total knee Arthroplasty in patients with end-stage renal disease: is it a safe procedure? J Arthroplasty. 2016;31(10):2152-5.

42. Ponnusamy KE, Jain A, Thakkar SC, Sterling RS, Skolasky RL, Khanuja HS. Inpatient mortality and morbidity for dialysis-dependent patients undergoing primary total hip or knee arthroplasty. J Bone Joint Surg (Am Vol). 2015;97(16):1326-32.

43. Yu YH, Chen WJ, Chen LH, Niu CC, Fu TS, Lai PL. Posterior instrumented lumbar spinal surgery in uremic patients under maintenance hemodialysis. Spine. 2011;36(8):660-6.

44. Ottesen TD, McLynn RP, Zogg CK, Shultz BN, Ondeck NT, Bovonratwet P, et al. Dialysis is an independent risk factor for perioperative adverse events, readmission, reoperation, and mortality for patients undergoing elective spine surgery. Spine J. 2018;18(11):2033-42.

45. Ottesen TD, Zogg CK, Haynes MS, Malpani R, Bellamkonda KS, Grauer JN. Dialysis patients undergoing Total knee Arthroplasty have significantly increased odds of perioperative adverse events independent of demographic and comorbidity factors. J Arthroplast. 2018;33(9):2827-34.

46. Hickson LJ, Farah WH, Johnson RL, Thorsteinsdottir B, Ubl DS, Yuan BJ, et al. Death and postoperative complications after hip fracture repair: Dialysis effect. Kidney International Reports. 2018;3(6):1294-303.

47. Inoue T, Mizutamari M, Fukuda K, Hatake K. Postoperative complications in Dialysis-dependent patients undergoing elective decompression surgery without fusion or instrumentation for degenerative cervical or lumbar lesions. Spine. 2018;43(17):1169-75.

48. Lin SJ, Chen TH, Kuo LT, Yu PA, Chen CL, Hsu WH. Effects of chronic kidney disease on Hemiarthroplasty outcomes for fragility hip fracture in diabetic patients: a Nationwide population-based observational study. J Arthroplasty. 2019.

49. Cancienne JM, Kew ME, Deasey MJ, Brockmeier SF, Werner BC. Dialysis dependence and modality impact complication rates after shoulder arthroplasty. J Shoulder Elb Surg. 2019;28(3):e71-e7.
50. Hibino M, Oshima H, Narita Y, Abe T, Mutsuga M, Fujimoto KL, et al. Early and late outcomes of thoracic aortic surgery in hemodialysis patients. Ann Thorac Surg. 2016;102(4):1282-8.

51. Lantis JC, Conte MS, Belkin M, Whittemore AD, Mannick JA, Donaldson MC. Infrainguinal bypass grafting in patients with end-stage renal disease: improving outcomes? J Vasc Surg. 2001;33(6):1171-8.

52. O'Hare AM, Feinglass J, Reiber GE, Rodriguez RA, Daley J, Khuri S, et al. Postoperative mortality after nontraumatic lower extremity amputation in patients with renal insufficiency. J Am Soc Nephrol. 2004;15(2):427-34.

53. Balceniuk MD, Zhao P, Ayers BC, Doyle AJ, Glocker RJ, Stoner MC. IP199. Association Between Distal Lower Extremity Bypass and Dialysis Status on Short-term Outcomes. J Vasc Surg. 2019;69(6):e164

54. Ambur V, Park P, Gaughan JP, Golarz S, Schmieder F, Van Bemmelen P, et al. The impact of chronic kidney disease on lower extremity bypass outcomes in patients with critical limb ischemia. J Vasc Surg. 2019;69(2):491-6.

55. Gajdos C, Hawn MT, Kile D, Henderson WG, Robinson T, McCarter M, et al. The risk of major elective vascular surgical procedures in patients with endstage renal disease. Ann Surg. 2013;257(4):766-73.

56. Hickson LJ, Rule AD, Thorsteinsdottir B, Shields RC, Porter IE, Fleming MD, et al. Predictors of early mortality and readmissions among dialysis patients undergoing lower extremity amputation. J Vasc Surg. 2018;68(5):1505-16.

57. Fornara P, Doehn C, Miglietti G, Fricke L, Steinhoff J, Sack K, et al. Laparoscopic nephrectomy: comparison of dialysis and non-dialysis patients. Nephrology Dialysis Transplantation. 1998;13(5):1221-5.

58. Schmitges J, Trinh QD, Sun M, Hansen J, Bianchi M, Jeldres C, et al. Higher perioperative morbidity and in-hospital mortality in patients with end-stage renal disease undergoing nephrectomy for non-metastatic kidney cancer: a population-based analysis. BJU Int. 2012;110(6B):E183-E90.

59. Yamashita K, Ito F, Nakazawa H. Perioperative outcomes of laparoscopic radical nephrectomy for renal cell carcinoma in patients with Dialysisdependent end-stage renal disease. Ther Apher Dial. 2012;16(3):254-9.

60. May D, Khaled D, Matrana MR, Bardot SF, Lata-Arias K, Canter D. Effect of the need for preoperative Dialysis on perioperative outcomes on patients undergoing laparoscopic nephrectomy: an analysis of the National Surgical Quality Improvement Program Database. Urology. 2019;124:154-9.

61. Nicholas GG, Bozorgnia M, Nastasee SA, Reed IJF. Infrainguinal bypass in patients with end-stage renal disease: survival and ambulation. Vasc Surg. 2000;34(2):147-56.

62. Rao A, Baldwin M, Cornwall J, Marin M, Faries P, Vouyouka A. Contemporary outcomes of surgical revascularization of the lower extremity in patients on dialysis. Journal of Vascular Surgery. 2017.

63. Thourani VH, Sarin EL, Kilgo PD, Lattouf OM, Puskas JD, Chen EP, et al. Short-and long-term outcomes in patients undergoing valve surgery with end-stage renal failure receiving chronic hemodialysis. J Thorac Cardiovasc Surg. 2012;144(1):117-23.

64. Sarnak MJ, Amann K, Bangalore S, Cavalcante JL, Charytan DM, Craig JC, et al. Chronic Kidney Disease and Coronary Artery Disease. $<$ span class= "subtitle" $><$ em $>$ JACC </em> State-of-the-Art Review</span>. 2019;74(14): 1823-38.

65. Berman SJ, Johnson EW, Nakatsu C, Alkan M, Chen R, LeDuc J. Burden of infection in patients with end-stage renal disease requiring long-term Dialysis. Clin Infect Dis. 2004;39(12):1747-53.

66. Doyle JF, Forni LG. Acute kidney injury: short-term and long-term effects. Crit Care. 2016;20(1):188.

67. Powe NR, Jaar B, Furth SL, Hermann J, Briggs W. Septicemia in dialysis patients: Incidence, risk factors, and prognosis. Kidney International. 55(3): 1081-90.

68. McDonald SP, Marshall MR, Johnson DW, Polkinghorne KR. Relationship between Dialysis modality and mortality. J Am Soc Nephrol. 2009;20(1):155-63.

69. Chertow GM, Johansen KL, Lew N, Lazarus JM, Lowrie EG. Vintage, nutritional status, and survival in hemodialysis patients. Kidney Int. 2000; 57(3):1176-81.

70. Mathew A, Devereaux P, O'hare A, Tonelli M, Thiessen-Philbrook H, Nevis I, et al. Chronic kidney disease and postoperative mortality: a systematic review and meta-analysis. Kidney Int. 2008;73(9):1069-81.

71. Palamuthusingam D, Johnson DW, Hawley CM, Pascoe E, Sivalingam P, Fahim M. Perioperative outcomes and risk assessment in dialysis patients: current knowledge and future directions 2019;49(6):702-710.

\section{Publisher's Note}

Springer Nature remains neutral with regard to jurisdictional claims in published maps and institutional affiliations. 